\title{
Manejo del paciente con fisura labio-palatina en Arica. Experiencia de 15 años
}

\author{
Management of cleft lip palate in Arica. 15 years experience
}

Emilio Godoy $R^{1,4}$, Alejandro Godoy $S^{2}$, Fernando Godoy $S^{2}$, Luis Monasterio $A^{3}$, Geldy Suazo $A^{1}$.

\begin{abstract}
RESUMEN
Introducción: Frente al alto número de pacientes con fisura labial y/o palatina ya existente en la ciudad de Arica y al incremento constante de nuevos casos, en 1987 decidimos abordar el problema desde un punto de vista multidisciplinario e interdisciplinario, integrando además la valiosa ayuda de los padres de los niños fisurados.

Objetivo: Mostrar nuestra experiencia y sus resultados parciales en el manejo multidisciplinario del paciente fisurado en la ciudad de Arica entre los años 1987 y 2002.

Material y método: Mediante la creación y puesta en práctica del "Programa de Atención del Paciente Fisurado", en el Hospital Juan Noé de Arica entre los años 1987 y 2002, se atienden 59 pacientes que siguen el programa.

Resultados: A modo de resultado preliminar se entrega la experiencia de 59 casos que siguieron el protocolo del programa.

Conclusión: El trabajo con el paciente fisurado, es un trabajo de largo aliento. Como factores de importancia se destacan el largo tiempo de seguimiento, el factor crecimiento y la observación atenta de la evolución en cada caso en particular. Resulta imprescindible el trabajo en equipo multidisciplinario, evaluando en conjunto cada caso, elaborando un claro y estructurado plan de acción con cada paciente, así como evaluar en forma periódica y en conjunto los resultados parciales.
\end{abstract}

Palabras clave: Fisura labial palatina, Labiopalatina, Otorrinolaringología, Arica.

\section{ABSTRACT}

Introduction: Due to the high prevalence of patients with cleft lip with or without cleft palate in the city of Arica Chile and the constant increase of new cases, in 1987 we decided to face the problem from a multidisciplinary and interdisciplinary point of view, incorporating the valuable help from the parents of the affected children.

Aim: To show our experience in the multidisciplinary manage of cleft lip-palate in the city of Arica during the years 1987 to 2002

\footnotetext{
Otorrinolaringólogo. Hospital Juan Noé, Arica.

2 Interno de Medicina. UFRO.

3 Cirujano Plástico Infantil. Clínica Alemana de Santiago.

4 Otorrinolaringólogo. Clínica Alemana Temuco.
} 
Material and method: By the creation and set up of the "Cleft Lip-Palate patient attention program", in the Juan Noé Hospital of Arica during the years 1987 to 2002, we follow 59 patients attending the program.

Results: Some preliminary results are displayed, according to the experience of 59 cases of patients attending the program.

Conclusion: Complete management of the clef lip patient is a long job. As important factors outstand age, growth and careful observation of evolution in every single case. Multidisciplinary team work is a must, evaluating each case together, developing a clear structured plan of action for each patient, as well as periodically evaluating partial results.

Key words: Cleft; Lip; Palate; Otolaryngology; Arica.

\section{INTRODUCCIÓN}

La fisura labio-palatina trae como consecuencia al paciente múltiples problemas en la alimentación, respiración nasal, audición, crecimiento facial, desarrollo dental, fonación, estéticos y psicológicos, cuyo grado varía en relación al compromiso labial, arcada dentaria, paladar y nariz.

En Chile la incidencia de fisura labio-palatina es en general de 1 por cada 580 nacidos vivos ${ }^{1}$. Esta patología es más frecuente en las razas asiáticas e indígenas de América. Alrededor de $66 \%$ de los pacientes tienen fisura de labio y paladar, $23 \%$ fisura aislada de paladar y $11 \%$ fisura aislada de labio². La incidencia de fisura labio-palatina en la ciudad de Arica es aún mayor, llegando en el año 1989 a 1 por cada 300 nacidos vivos (datos obtenidos del libro de partos del Hospital Juan Noé de Arica el año 1989).

La ciudad de Arica, situada a $2.000 \mathrm{Km}$. de la capital, se encuentra aislada geográficamente del país y de los centros médicos especializados, de manera que se deben realizar grandes esfuerzos para tratar adecuadamente las distintas patologías de relativa y alta complejidad.

Al momento de hacernos cargo de estos pacientes en 1986, nos encontramos con un número indeterminado de pacientes con fisura labiopalatina de todas las edades, desde niños a adultos, con distintos tipos de tratamiento, distintas variedades de fisuras labio-palatinas y distintos resultados. Sumándose a esto una incidencia de entre 6 a 7 pacientes nuevos por año.

La comuna de Arica es una comunidad casi $100 \%$ urbana, de manera que hace posible el tener una adherencia relativamente buena en los planes de salud a largo plazo.
Con el fin de lograr entonces un adecuado tratamiento integral del paciente fisurado, el año 1987 comenzamos a formar un equipo multidisciplinario, tanto del sistema público como del sistema privado, integrando además a los padres de niños fisurados.

De trascendental importancia para nuestro objetivo resultó la ayuda del Dr. Luis Monasterio Aljaro, médico cirujano plástico infantil y director de la Fundación Gantz, de quien obtuvimos conocimientos, capacitación y ayuda técnica directa en los casos más complejos, operando con nosotros en nuestro Hospital.

\section{OBJETIVO}

El presente trabajo, tiene como objetivos: a) Dar a conocer nuestra experiencia en el manejo multidisciplinario del paciente fisurado en la ciudad de Arica entre los años 1987 y 2002. b) Mostrar los resultados preliminares del "Programa de Atención del Paciente Fisurado", dirigido por el Servicio de Otorrinolaringología del Hospital Juan Noé de Arica.

\section{MATERIAL Y MÉTODO}

Para definir y organizar la labor, se creó el "Programa de Atención del Paciente Fisurado", en el cual se definieron los profesionales integrantes, el universo de pacientes y los objetivos perseguidos, el que, en forma resumida, se muestra a continuación: 


\section{Programa de Atención del Paciente Fisurado}

\section{Objetivos}

1. Dar total atención profesional, médico-quirúrgica, fonoaudiológica, dental, otorrinolaringológica, social y psicológica, tanto al paciente como a su familia.

2. Conocer las estadísticas de la patología en la ciudad.

3. Apoyo y educación continua a los padres de los niños fisurados.

4. Hacer conciencia en la comunidad y conminarla a prestar ayuda y apoyo.

\section{Grupo de profesionales y actividades:}

- Otorrinolaringólogo: Encargado de la coordinación del programa, realizando además cirugías de labio, paladar, oído y nariz.

- Cirujano Plástico Infantil: Evaluación de casos, realiza cirugía de casos de mayor complejidad y revisiones.

- Odontólogo: Realiza toda la operatoria dental correspondiente.

- Ortodoncistas: Tratamiento de ortodoncia.

- Fonoaudiólogas: Evaluación y tratamiento de audición y lenguaje.

- Pediatra: Evaluación pediátrica general y especial de la patología.

- Asistente social: Enlace entre el grupo de padres de niños fisurados y el equipo.

- Enfermera: Manejo de enfermería del paciente hospitalizado

- Psicóloga: Ayuda y apoyo a los pacientes y su familia en casos requeridos.

Este grupo de profesionales se constituyó aunando fuerzas de profesionales de nuestro hospital y con ayuda de dentistas, ortodoncistas y fonoaudióloga del extrasistema, quienes ofrecieron su ayuda en forma desinteresada.

\section{Dinámica de trabajo:}

El grupo se reunía en forma mensual para analizar cada caso, proponer una pauta de trabajo con cada paciente y analizar los resultados del programa en general. Los acuerdos de cada reunión quedaban registrados en un acta.

Además de la ficha clínica correspondiente a la institución y de la propia del equipo, cada paciente tenía una ficha, la que va con el paciente (cuader- no). En él, cada profesional anotaba el trabajo que se estaba realizando, de manera que siempre esté disponible la información de los avances de cada profesional, adhesión al programa y progreso en cronograma del paciente.

\section{Cronograma ideal de atención del paciente}

Este contempla los siguientes pasos:

1. Nacimiento: Visita del pediatra, otorrinolaringólogo y padres de niños fisurados. Personalmente al momento de examinar al paciente, se explica a los padres en lenguaje sencillo, la lesión, sus implicancias en sus diversos aspectos, además se enseña la cronología del tratamiento. Se deja claramente establecido que están en un programa multidisciplinario. Se responden las preguntas de los padres y en conjunto se les presenta a dos madres de niños fisurados, las cuales transmiten sus experiencias, logrando de esta manera tranquilizar a los nuevos padres del niño fisurado recién nacido. Los padres de estos nuevos pacientes, reciben además un folleto con un resumen de la patología, cronograma de tratamiento e indicaciones generales. Quedando contactados con la agrupación de padres y con su médico a cargo (en nuestra experiencia, el otorrinolaringólogo).

2. Tres meses: Cirugía del labio y nariz.

3. Un año: Cierre del paladar blando.

4. Dos años: Cierre del paladar duro.

5. Un año en adelante: Fonoaudiología.

6. Seis años en adelante: Operatoria dental y ortodoncia.

7. Diez y seis años en adelante: rinoplastia secundaria o rinoseptoplastia.

\section{RESULTADOS}

Debido al bajo número de pacientes tratados a la fecha como para analizar cada técnica en cada distinta cirugía realizada, el presente trabajo pretende sólo mostrar nuestra experiencia en la manera de enfrentar esta patología y el manejo de ella, en una primera instancia desde el punto de vista general, destacando como condición necesaria para obtener buenos resultados el trabajar en equipo. En segundo término se muestran resultados parciales a modo de ejemplo, del preoperatorio 
y posoperatorio final de dos técnicas quirúrgicas de cierre de fisura labial unilateral primaria.

Dentro del periodo evaluado (1987-2002), se incluyen 59 pacientes cuyas edades van desde recién nacidos hasta los 18 años. De estos pacientes, los menores de 2 años eran los únicos sin ningún tipo de tratamiento, con excepción de un niño de 6 años con fisura labio-palatina total unilateral sin tratamiento. Todos los niños mayores de 6 años ya habían recibido tratamiento y se encontraban en distintas etapas y con variados resultados. De esta manera nos enfrentamos a resolver casos de variadas características y complejidades.

\section{Distribución por edad}

El mayor número de pacientes se encuentra agrupado en los 2 primeros años de vida, el mayor de los pacientes tenía 18 años (Tabla 1).

Tabla 1. Distribución por edad

\begin{tabular}{|lc|}
\hline Edad & Pacientes \\
\hline 1 a 2 & 19 \\
3 a 4 & 14 \\
4 a 5 & 9 \\
5 a 6 & 7 \\
7 a 10 & 5 \\
11 a 15 & 3 \\
16 y más & 2 \\
\hline Total & 59 \\
\hline
\end{tabular}

\section{Distribución por sexo}

Se encontró $68 \%$ de sexo masculino y $32 \%$ de sexo femenino (Tabla 2).

\section{Tipo de lesión}

Entre los pacientes evaluados sin tratamiento previo, la lesión más frecuente resultó ser la fisura labio-palatina unilateral total, seguida por la fisura de paladar aislada (Tabla 3).

\section{Resultados quirúrgicos}

Los resultados obtenidos se presentan en forma de ejemplos de dos diferentes técnicas y en forma visual considerando pre y poscirugía.

En los distintos estados de los pacientes fue necesario realizar diversas técnicas quirúrgicas. De ellas destacamos: adhesión primaria de labio, queiloplastia primaria de labio unilateral con técnica de Millard ${ }^{3}$, quieloplastia primaria con técnica de Skoog ${ }^{4}$, queiloplastia de fisura labial bilateral con técnica de Millard, cierre de paladar blando, cierre de paladar duro, faringoplastias, queiloplastias secundarias, septoplastias, rinoseptoplastias, cierre de fístulas oro-nasales y vestíbulo-nasales, colocación de tubos de ventilación timpánica.

Tabla 2. Distribución por sexo

\begin{tabular}{|lc|}
\hline Sexo & Número \\
\hline Masculino & 40 \\
Femenino & 19 \\
\hline
\end{tabular}

Tabla 3. Tipo de lesión

\begin{tabular}{|lrr|}
\hline Lesión & $\%$ & $\mathrm{~N}^{0}$ \\
\hline Fisura labio-palatina total unilateral & 58,93 & 33 \\
Fisura paladar blando & 17,86 & 10 \\
Fisura labial unilateral 2/3 & 3,57 & 2 \\
Fisura labial unilateral 1/3 & 8,93 & 5 \\
Fisura labial 2/3 + fisura paladar total unilateral & 3,57 & 2 \\
Fisura labial y alveolar total & 3,57 & 2 \\
Fisura labio-palatina total bilateral & 3,57 & 2 \\
\hline
\end{tabular}


En el presente trabajo se ilustran algunos casos de pacientes con fisura labial unilateral, en los cuales se aplicaron dos distintas técnicas de queiloplastia primaria en relación al grado de separación de la fisura.

\section{DISCUSIÓN}

El manejo de la fisura labio-palatina requiere necesariamente la concurrencia de un equipo multidisciplinario para poder resolver adecuadamente los múltiples aspectos que conlleva ${ }^{5}$. Cada paciente requiere tratamientos que se prolongan en el tiempo y que están en relación al crecimiento y desarrollo, por lo tanto el seguimiento y los resultados son a largo plazo.

En los casos de fisura labial unilateral, dependiendo del grado de la fisura labial y de la diferen- cia en altura entre el segmento fisurado y el sano, la técnica empleada es distinta. En aquellos en que la diferencia de altura no es mayor de $4 \mathrm{~mm}$ y la separación de los segmentos es poca se realiza la técnica de rotación y avance de Millard ${ }^{3}$, como se ilustra en las Figuras 1, 2, 4 y 5. En los casos con diferencia de altura mayor a $4 \mathrm{~mm}$ y/o existe una separación amplia, se prefiere la técnica de colgajos triangulares, en este caso la técnica de Skoog, como se ilustra en las Figuras 3, 6,7 y $8^{4}$. En los casos bilaterales se utiliza la técnica de Millard.

Dado que uno de los estigmas estéticos más notorios en estos pacientes es el aspecto de la nariz, decidimos realizar en nuestros pacientes en el mismo momento de la cirugía del labio, la rinoplastia primaria. Se procede liberando los cartílagos alares en colgajos bipediculados, ampliando la liberación de la escotadura piriforme,

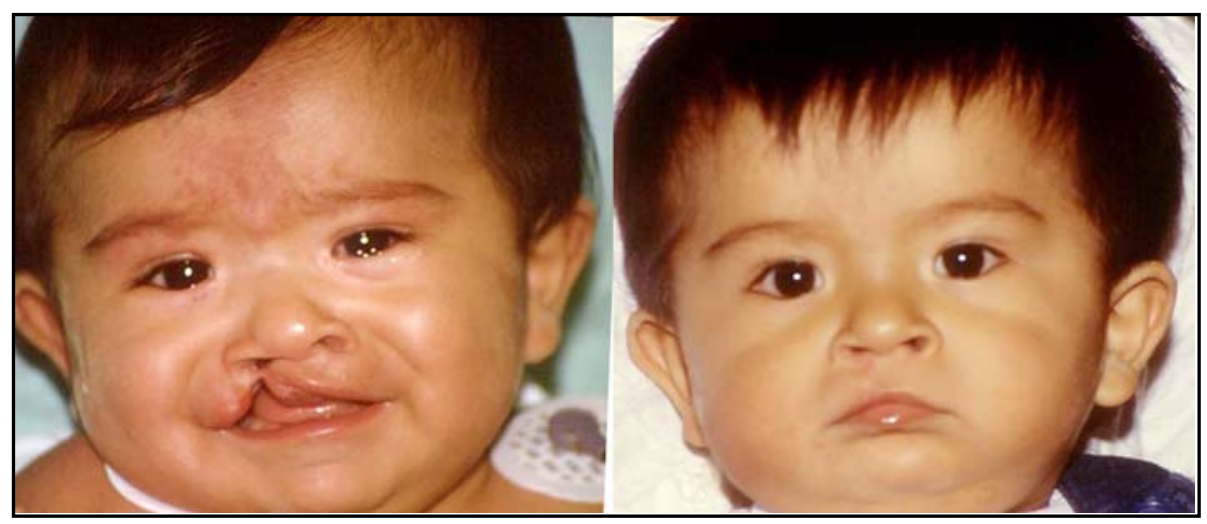

Figura 1. Queiloplastia con técnica de rotación y avance de Millard.

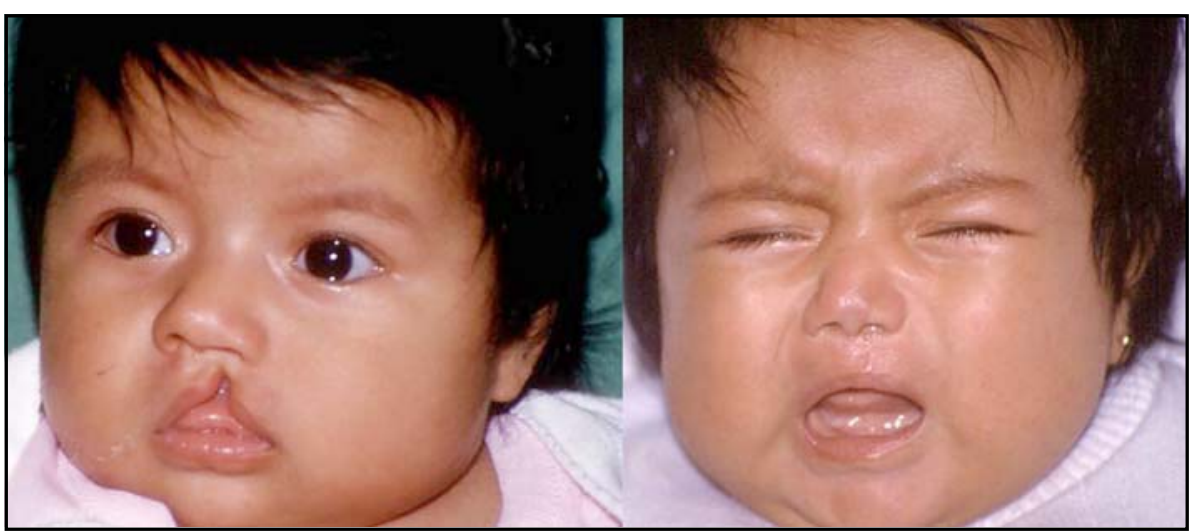

Figura 2. Queiloplastia con técnica de rotación y avance de Millard. 


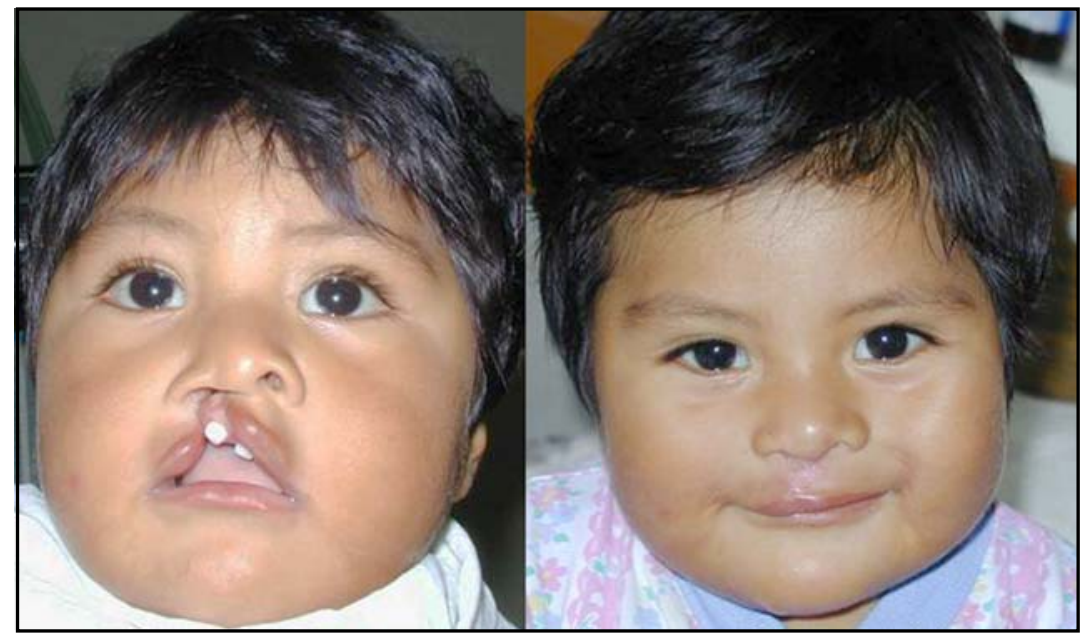

Figura 3. Queiloplastia con técnica de colgajos triangulares de Skoog.

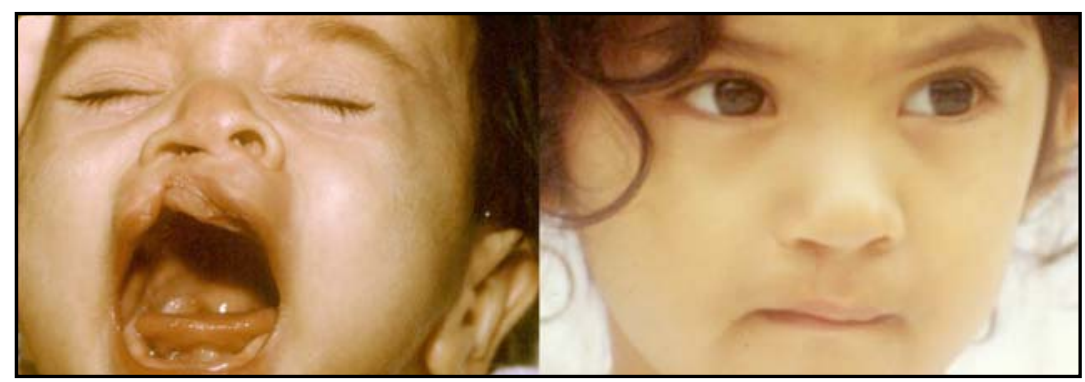

Figura 4. Queiloplastia con técnica de rotación y avance de Millard.

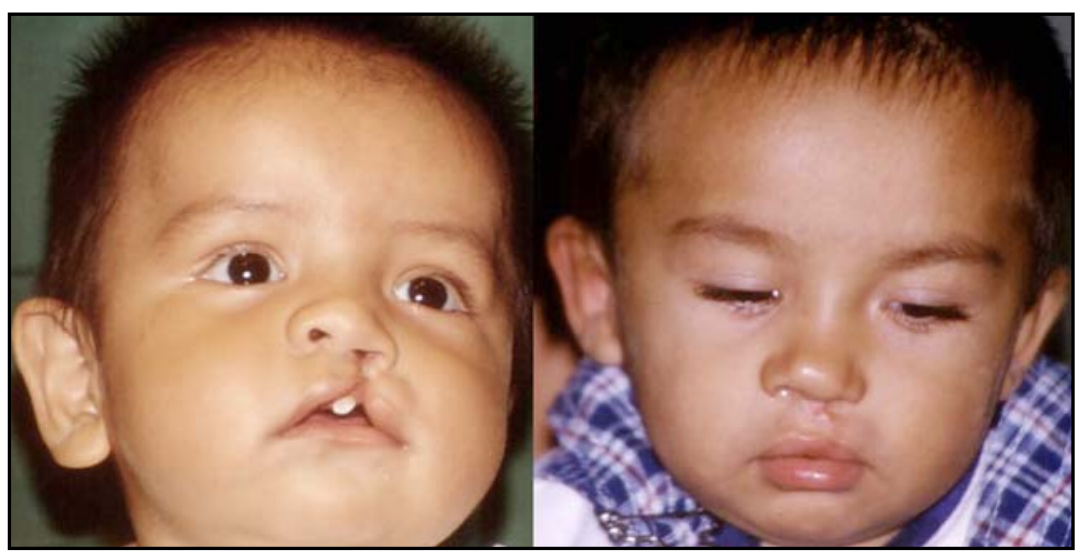

Figura 5. Queiloplastia con técnica de rotación y avance de Millard.

colocación de puntos interdomales y de suspensión del alar del lado fisurado, hasta lograr una mayor simetría nasal ${ }^{8}$.

El objetivo más importante del tratamiento de la fisura palatina es lograr una voz normal, de manera que idealmente procuramos establecer lo más rápidamente posible la anatomía del paladar, a pesar de las consideraciones del crecimiento facial. Las alteraciones de crecimiento maxilar por cierre precoz se pueden tratar con ortodoncia, cirugía 


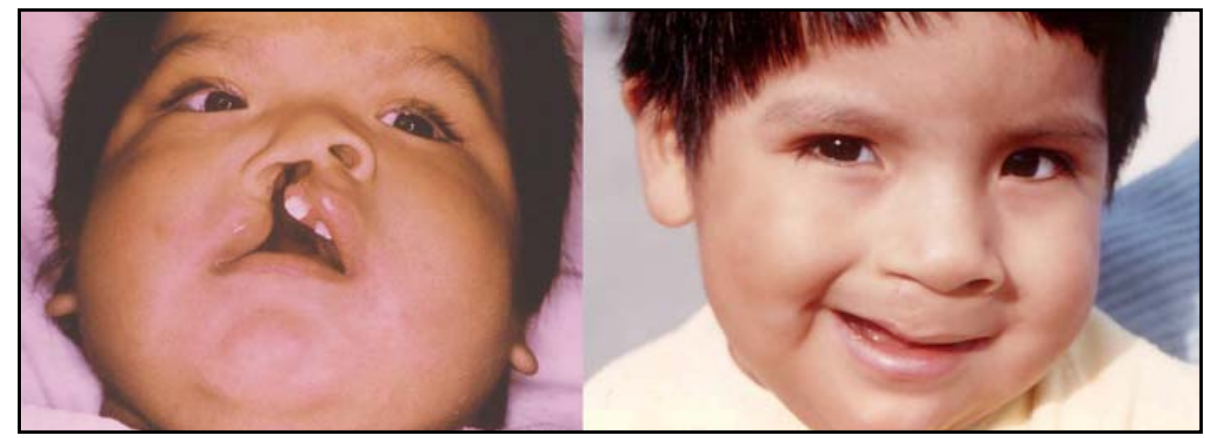

Figura 6. Queiloplastia con técnica de colgajos triangulares de Skoog.

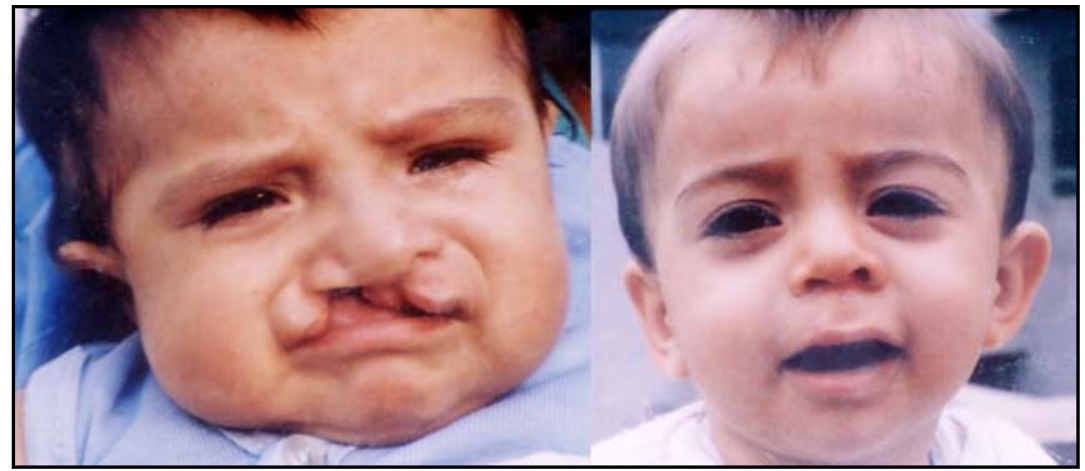

Figura 7. Queiloplastia con técnica de colgajos triangulares de Skoog.

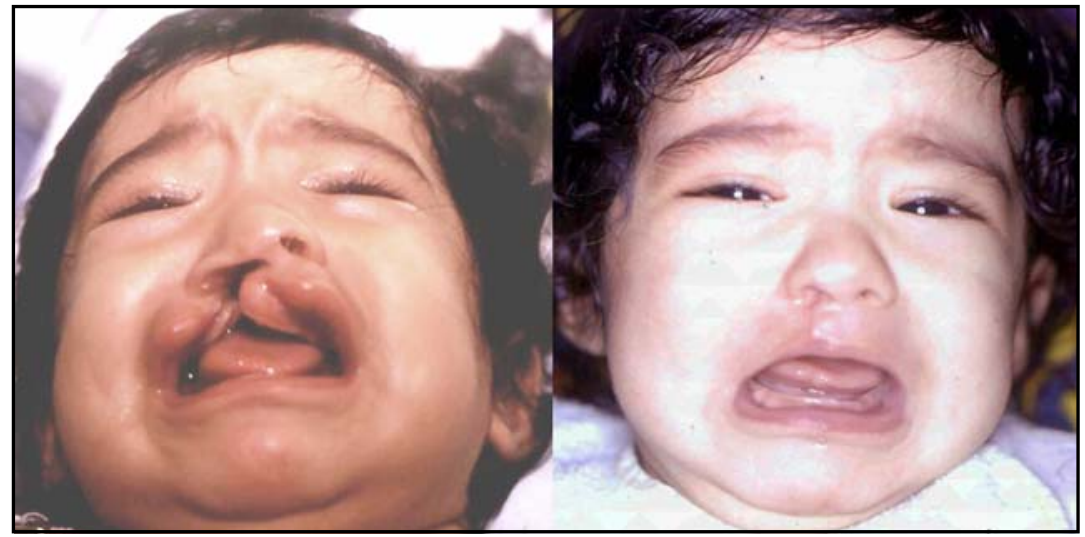

Figura 8. Queiloplastia con técnica de colgajos triangulares de Skoog.

ortognática 0 distracción ósea, pero las secuelas foniátricas son irreversibles ${ }^{6}$.

Se realiza el cierre del velo palatino con plastia intravelar de Kriens restableciendo la anatomía normal del Elevador del Velo ${ }^{7}$, además de prolongar el velo con la técnica de Sanvenero-Rosselli ${ }^{6}$.
En casos de gran separación se realizan incisiones de descarga y en casos requeridos la sección del músculo Tensor del Velo o la luxación de su tendón del Hamulus (6, Pág. 372).

Nuestra limitada experiencia no nos permite de momento elaborar conclusiones significativas, de 
manera que el aporte de esta publicación es sólo dar a conocer el trabajo realizado y mostrar nuestros resultados parciales de algunas cirugías de fisura labial unilateral.

\section{CONCLUSIONES}

El trabajo con el paciente fisurado, es un trabajo de largo aliento, en donde los años, el factor crecimiento y la observación atenta de la evolución de cada paciente nos va aportando para obtener resultados satisfactorios.

Resulta imprescindible el trabajo en equipo multidisciplinario, evaluando en conjunto cada caso, elaborando un claro y estructurado plan de acción con cada paciente, así como evaluar en forma periódica y en conjunto los resultados parciales.

En relación a los casos ilustrados de fisura unilateral, podemos concluir que se obtiene un mejor resultado estético con técnica de rotación y avance de Millard en los casos con poca separación del segmento fisurado. En los casos de amplia separación la técnica de colgajos triangulares de Skoog presenta los mejores resultados.

También concluimos que se debe intervenir primariamente, en el mismo tiempo del cierre del labio, la nariz con técnica definida, teniendo como objetivo el lograr la mayor simetría nasal posible, con lo cual el estigma de la punta nasal caída hacia el lado fisurado desaparece.

\section{Agradecimientos}

Expresamos nuestro gran reconocimiento al Dr. Luis Monasterio Aljaro, Médico Cirujano Plástico de la Clínica Alemana de Santiago y Director de la Fundación Gantz. Su apoyo constante y enseñanza ha hecho posible nuestro objetivo y ha contribuido a mejorar la salud y calidad de vida de los pacientes fisurados de la ciudad de Arica. También se agradece al restante grupo que ha participado en el programa: Dr. Carlos Crignola S., Dr. Luis Guajardo C., Dr. Juan Campusano A., Fga. Elizabeth Guajardo C, Fga. María Herrera G.

\section{BIBLIOGRAFÍA}

1. Nazer J, Hubner me, Catalán J, Cifuentes l. Incidence of cleft lip and palate in the University of Chile Maternity Hospital and in Chilean maternities participating in the Latin American Collaborative study of congenital malformations (ECLAMC). Rev Méd Chile 2001; 129(3): 285-93.

2. Ford A.Tratamiento actual de las fisuras labiopalatinas. Rev Med Clin Condes 2004; 15(1): 3-11.

3. Millard D Ralph. Cleft craft : the evolution of its surgery. D. Ralph Millard, Jr. $1^{\text {st }}$ ed. Little, Brown, Boston: 1976.

4. Skoog T. Cirugía plástica: nuevos métodos y aportaciones. Salvat Editores, 1976.

5. Vlastos I, Koudoumnakis E, Houlakis M, Nasika M,Griva M, Stylogianni E. Cleft lip and palate treatment of 530 children over a decade in a single centre. Int J Pediatr Otorhinolaryngol 2009; 73: 993-7.

6. Monasterio L. Tratamiento interdisciplinario de las fisuras labio-palatinas. Cir Plást Iberolatinoam [online]. 2009, vol.35, n.1 [citado 2010-05-08], pp. 91-91. Disponible en: http://scielo.isciii.es/scielo.php?pid=S0376$78922009000100017 \&$ \&cript=sci_arttext\&tlng=pt.

7. KRIENS OB. Fundamental Anatomic Findings for an Intravelar Veloplasty. Cleft Palate J Vol. 7, January, 1970.

8. Trott JA, Mohan N. A preliminary report on one stage open tip rhinoplaty at the time of lip repair in bilateral cleft lip and palate: the Alor Setar experience. Br J Plast Surg 1993; 46(3): 215-22.

9. Mcheik J, Sfalli P, Bondonny J, Levard G. Early repair for infants with cleft lip and nose. Int $J$ Pediatr Otorhinolaryngol 2006; 70: 1785-90.

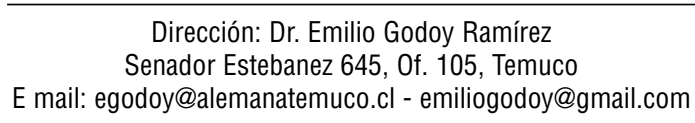

Dirección: Dr. Emilio Godoy Ramírez

E mail: egodoy@alemanatemuco.cl - emiliogodoy@gmail.com 